\title{
Higher-order Genome Organization in Human Disease
}

\author{
Tom Misteli \\ National Cancer Institute, NIH, Bethesda, Maryland 20892 \\ Correspondence: mistelit@mail.nih.gov
}

Genomes are organized into complex higher-order structures by folding of the DNA into chromatin fibers, chromosome domains, and ultimately chromosomes. The higher-order organization of genomes is functionally important for gene regulation and control of gene expression programs. Defects in how chromatin is globally organized are relevant for physiological and pathological processes. Mutations and transcriptional misregulation of several global genome organizers are linked to human diseases and global alterations in chromatin structure are emerging as key players in maintenance of genome stability, aging, and the formation of cancer translocations.

\begin{abstract}
Cenomes in their native state are folded $\checkmark i n t o$ complex higher-order structures. Hierarchical folding of DNA gives rise to chromatin fibers, chromosomes domains, and eventually chromosomes (reviewed in Felsenfeld and Groudine 2003; Belmont 2006; Woodcock 2006). At the lowest level, DNA is wrapped around an octamer of core histone proteins, which are the primary architectural elements of the chromatin fiber, to form a nucleosome. Multiple nucleosomes are linked by stretches of DNA, often occupied by a linker histone, into a beads-on-a string fiber of $\sim 10 \mathrm{~nm}$ in diameter. This primary fiber is then further compacted onto itself to form higher-order fibers of various diameters, although their precise geometry in vivo is unknown (reviewed in Belmont 2006; Woodcock 2006). At the next level of organization, the chromatin fiber folds into subchromosomal domains of $\sim 1 \mathrm{Mb}$ in
\end{abstract}

size (reviewed in Cremer and Cremer 2001; Cremer et al. 2006). These subchromosomal domains in turn are folded to give rise to an interphase chromosome. In mammalian cells, the degree of fiber compaction from naked DNA to a chromosome is estimated to be on the order of $\sim 10,000$-fold (reviewed in Belmont 2006).

During interphase, chromosomes exist as chromosome territories (reviewed in Cremer and Cremer 2001; Meaburn and Misteli 2007; Misteli 2007). A chromosome territory is defined as the nuclear space taken up by the DNA of a given chromosome. The term "territory" refers to the fact that the occupied space is compact, typically roughly ovoid in shape, with a volume of about $2-3 \mu \mathrm{m}$ in diameter (reviewed in Cremer and Cremer 2001; Meaburn and Misteli 2007; Misteli 2007). The internal structure of chromosome territories is

Editors: Tom Misteli and David L. Spector

Additional Perspectives on The Nucleus available at www.cshperspectives.org

Copyright (C) 2010 Cold Spring Harbor Laboratory Press; all rights reserved; doi: 10.1101/cshperspect.a000794

Cite this article as Cold Spring Harb Perspect Biol 2010;2:a000794 
poorly understood but likely consists of a highly interconnected and branched network of channels, which form between looping chromatin fibers (reviewed in Cremer and Cremer 2001; Meaburn and Misteli 2007; Misteli 2007). This relatively open structure allows access of gene regulatory factors into the interior of chromosome territories. Although chromosome territories are discrete structures within the nucleus, neighboring chromosomes can overlap considerably and chromatin loops from one territory can easily invade the body of the neighboring territory (Visser et al. 2000; Branco and Pombo 2006).

The higher-order organization of the genome into chromatin fibers and chromosomes is now known to critically contribute to gene regulation (reviewed in Fraser and Bickmore 2007; Lanctot et al. 2007). It is therefore not surprising that defects in higher-order chromatin and chromosome organization cause disease. There is an increasing list of diseases in which changes in histone modifications have been documented, although it is often not clear whether these alterations are a cause or merely a side-effect of the disease process. Furthermore, although many disease-related epigenetic changes have implicitly been assumed to result in changes in higher-order chromatin structure, it is often not clear whether the observed disease phenotypes are because of structural chromatin defects or because of altered gene expression caused by local changes in histone modifications at particular genes. This article focuses primarily on disease mechanisms that involve bona-fide structural defects in chromatin.

\section{ARCHITECTURAL CHROMATIN PROTEINS IN DISEASE}

The higher-order structure of chromatin is mediated by a multitude of architectural chromatin proteins (reviewed in Woodcock 2006; Hock et al. 2007; Fanti and Pimpinelli 2008; Phillips and Corces 2009). Although some of these proteins, such as linker histones, highmobility group proteins, and heterochromatin protein 1, decorate the chromatin fiber extensively, others associate with the fiber only locally and are often involved in formation of chromatin loops. Looping is a prominent and powerful mechanism of gene regulation because loops can bring together distantly located genome regions and in this way lead to physical proximity of a regulatory sequence and its target (reviewed in Misteli 2007; Phillips and Corces 2009). Several key architectural chromatin proteins, many involved in looping, have been implicated in disease.

\section{SATB1}

SATB1 (Special AT-rich binding protein 1) is a thymocyte specific DNA-binding protein that was initially characterized because of its propensity to bind to AT-rich sequences that unwind in response to superhelical tension (Dickinson et al. 1992). This unique binding property already hinted at a potential structural role for SATB1 in higher-order genome organization. Such a role was confirmed by the finding that SATB1 forms a "cagelike" matrix within the nucleus of thymocytes and serves to anchor, via loop formation, a large number of genes whose activity is tightly regulated during T-cell differentiation (Cai et al. 2003). Regulation of several T-cell differentiation genes is dependent on SATB1 and its loss leads to dramatic differentiation defects. Although some of these effects are likely because of a role of SATB1 in recruiting histone modifying activities and chromatin remodeling complexes, some of its regulatory function has been attributed to its ability to promote formation of higher-order chromatin loops (Cai et al. 2006). For example, the activation of the Thelper-cell cytokine locus 2 during T-cell differentiation requires the superposition of an upstream Locus Control Region (LCR) with its promoter region. SATB1 is required for this looping event and is found associated with the locus at what is likely the base of the loop (Cai et al. 2006). An unresolved issue is whether SATB1 acts itself as a structural scaffold for these loops or as an adaptor to an underlying structural component.

SATB1 appears to be a critical player in cancer. The expression level of SATB1 correlates with poor prognosis in breast cancer and its 
expression induces aggressive growth of breast cells in vitro, whereas its depletion reverses cancer metastasis in animal models (Han et al. 2008). In metastatic breast cancer cells, SATB1 regulates a large number of genes predominantly involved in cell adhesion, cellular signaling, and cell-cycle regulation. SATB1 binds near many of these genes and its depletion leads to changes in their epigenetic makeup (Han et al. 2008). An attractive, although at present unproven, model is that SATB1 acts as a structural platform providing a base for chromatin loops and in this way tethers its target genes into a shared regulatory environment. Given that SATB1 is only expressed at relatively low levels in breast tissue, it will be interesting to determine whether the protein plays a similar role in other tumor types and whether it affects the same set of genes in other tumors. There are currently no reported mutations in SATB1 in cancer patients and it will be important to uncover the mechanism by which SATB1 is misregulated in cancer.

\section{CTCF}

CTCF (CCCTC-binding factor) is a zinc-finger protein with a central, evolutionarily highly conserved, DNA-binding domain (reviewed in Phillips and Corces 2009). The protein is widely expressed, although levels and localization within the nucleus are tissue- and cell-type specific. Both loss of CTCF as well as its overexpression have severe and global effects on gene expression profiles and lead to physiological defects in development and differentiation. Increasing evidence suggests that CTCT acts mainly as an insulator protein blocking the action of enhancers on a gene target or to buffer genes from adjacent regulatory sequences (reviewed in Phillips and Corces 2009). Various observations suggest that CTCF exerts this insulator function via chromatin looping. CTCF forms homodimers and multimers on chromatin and CTCF-mediated chromatin loops have been shown for various genes including mouse $\beta$-globin, human MHC class II, and the imprinted H19/Igf2 locus (Kurukuti et al. 2006; Splinter et al. 2006; Majumder et al.
2008). Because of its ability to readily form loops and because CTCF is widely bound throughout the genome with between 15,000-25,000 binding sites (Xie et al. 2005; Heintzman et al. 2009), it is a strong candidate for a global genome organizer (reviewed in Phillips and Corces 2009). This role is particularly strongly supported by the finding that CTCF can also mediate interchromosomal interactions. Genomewide studies have indicated that the imprinted H19/Ig2 locus on human chromosome 11 physically interacts with regulatory elements on other chromosomes and that these interchromosomal interactions are critical for efficient imprinting (Ling et al. 2006; Zhao et al. 2006). CTCF associates preferentially with the repressed, maternal allele of H19/Igf2 in a DNA methylation-dependent manner and most of the interchromosomal interactions occur at the maternal allele and are dependent on CTCF, suggesting that the protein plays a key role in its repression (Yoon et al. 2007; Li et al. 2008). It thus appears that CTCF acts as a global genome organizer by forming intrachromosomal loops and interchromosomal interactions.

CTCF has been implicated in various diseases. Trinucleotide repeat expansion diseases including Huntington's disease, fragile X mental retardation, and myotonic dystrophy are caused by excessive lengthening of microsatellite repeat sequences (reviewed in Orr and Zoghbi 2007). For example, although a healthy individual has a stretch of fewer than 27 CAG repeats in their HTT gene, a Huntington's disease patient has typically more than 35 . The trigger for repeat expansion is unknown. Recent evidence suggests that CTCF might contribute to generating fragile sites within repeats, thus facilitating their expansion (Libby et al. 2008). Mutation of a CTCF-binding site near a repeat leads to increased genomic instability and increased repeat length, similar to that seen in disease situations. Although not tested, it is possible that interference with CTCF binding, either by mutation of its target site or mutations in an interacting partner, may contribute to trinucleotide repeat diseases (Libby et al. 2008). Defects in CTCF, and other genome organizers, 
may also play a key role in destabilizing expanded microsatellite repeats in other trinucleotide repeat diseases (reviewed in Dion and Wilson 2009).

CTCF has also been suggested to play a role in two human syndromes, Silver-Russell (SRS) and Beckwith-Wiedeman Syndrome (BWS) (Sparago et al. 2004; Eggermann et al. 2008). BWS is a developmental disorder with variable clinical symptoms including increased frequency of tumors, macroglossia, and overgrowth. It is caused by aberrant activation of the normally maternally imprinted H19/Igf2 locus on chromosome 11. Several sporadic and familial mutations have been characterized in the H19/Igf2 region, which lead to the loss of CTCF binding sites, directly implicating CTCF in the disease (Sparago et al. 2004; Eggermann et al. 2008). Similarly, in SRS, a developmental disorder characterized by severe growth retardation and body asymmetry caused by activation of the paternal allele of Igf2, mutations in CTCF binding sites have been identified (Schönherr et al. 2008).

CTCF has also been implicated in cancer (Witcher and Emerson 2009). The tumor suppressor p16 is often up-regulated in cancer cells. This up-regulation coincides with a loss of the chromatin boundary definition around the locus leading to its activation and loss of chromatin structure in the vicinity of the locus (Witcher and Emerson 2009). CTCF associates with the boundaries of the transcriptionally active p16 gene, but not its inactive form, and p16 activity correlates with CTCF binding. In contrast, reduction of CTCF nearly ablates p16 expression (Witcher and Emerson 2009). Interestingly, the extent of CTCF binding in cancer cells is modulated by methylation of its binding site and cancer cells are well know to have global methylation defects. Although these observations strongly point to a role of CTCF in regulation of the tumor suppressor p16, other cancer relevant genes appear to be regulated in a similar fashion because silencing of the tumor suppressors RASSF1A and CDH1 also correlate with CTCF binding (Witcher and Emerson 2009). These observations allow for the possibility that CTCF plays a critical role in establishing and maintaining the higher-order chromatin structure around p16 and other tumor suppressor genes and in this way contributes to tumor prevention (Witcher and Emerson 2009). Whether mutations in CTCF have an oncogenic effect is unknown.

\section{Cohesin}

Cohesin is a multiprotein complex involved in establishment and maintenance of pairing of sister chromatids during DNA replication and into mitosis (reviewed in McNairn and Gerton 2008; Peters et al. 2008). The evolutionarily conserved complex consists of four core proteins, which are thought to form a ring around the replicated chromatids holding them together, and several accessory proteins involved in loading and maintenance of the complex (reviewed in McNairn and Gerton 2008; Peters et al. 2008). Although the traditional role of cohesin is in holding chromatids together, alternate roles are emerging. Cohesin is expressed at robust levels in postmitotic cells that do not have a requirement for chromatid cohesion. Furthermore, genome-wide mapping studies in several species show nonrandom association of cohesin with the genome and its distribution is responsive to ongoing transcription, suggesting its involvement in higher-order chromosome organization. In Saccharomyces cerevisiae cohesin is predominantly associated with intergenic regions and binds near convergently transcribed genes, as it does in Schizosaccharomyces pombe (Glynn et al. 2004; Lengronne et al. 2004; D'Ambrosio et al. 2008; Schmidt et al. 2009). In mammalian cells, cohesin colocalizes strikingly with CTCF in insulator regions (Parelho et al. 2008; Wendt et al. 2008) and in Drosophila melanogaster, the complex is generally found near highly transcribed regions of the genome (Misulovin et al. 2008). Further evidence for a role of cohesion in genome organization comes from the finding that a mutation in the cohesion subunit $\mathrm{Smcl}$ leads to loss of boundary function at the silent mating type locus in yeast and mutations in the Scc2/Nipped-B subunit leads to promoter-enhancer defects in D. melanogaster (Donze et al. 1999; Rollins et al. 1999). 
Mutations in cohesin components are the cause of two major multisystem developmental disorders, Cornelia de Lange Syndrome (CLS) and Roberts Syndrome (RS) (Krantz et al. 2004; Tonkin et al. 2004; Vega et al. 2005). CLS is a dominant disorder with severe growth defects and mental retardation; RS is a rare recessive disorder characterized by pre- and postnatal growth deficiency and mental retardation. However, patient cells do not show chromatin cohesion or segregation defects, suggesting that the disease symptoms are related to the noncohesion functions of the cohesin complex (reviewed in Dorsett and Krantz 2009). A likely scenario is that mutations in cohesin components weaken the complex's association with target sites throughout the genome and in this way interfere with its boundary and insulator function, possibly by cooperating with CTCF (reviewed in McNairn and Gerton 2008; Dorsett and Krantz 2009). Failure to properly define gene boundaries is predicted to result in widespread misexpression of genes and depletion of cohesin in human cells indeed leads to altered expression of several hundred genes (Wendt et al. 2008). Interestingly, cohesin is increasingly implicated in development via control of several developmentally regulated genes, possibly explaining the pre- and postnatal growth and development defects typically seen in CLS and RS (reviewed in Dorsett 2009).

\section{C-MYC}

C-MYC is one of the prototypical oncogenes. It encodes a basic helix-loop-helix zipper transcription factor and plays a role in numerous critical events in cancer including proliferation, cell cycle regulation, and apoptosis (reviewed in Eilers and Eisenman 2008). It has generally been assumed that c-myc exerts its function by acting on a few selected target genes whose misregulation would largely explain the oncogene's detrimental effects (reviewed in Eilers and Eisenman 2008). However, genome-wide mapping of c-myc binding sites has revealed that rather than binding to a few target genes, $\mathrm{c}$-myc binds to as many as $15 \%$ of human genes and also associates with intergenic regions, suggesting other functions for c-myc (Knoepfler 2007; reviewed in Eilers and Eisenman 2008). Several other observations also suggest that c-myc might have a more global function in higherorder chromatin structure. In knockout cells, neuronal stem cells show striking defects in nuclear architecture and chromatin organization including reduced nuclear size, aberrant nuclear morphology, and wide-spread chromatin condensation (Knoepfler et al. 2002; Knoepfler et al. 2006). Importantly, these changes appear to be independent of functional defects on proliferation and apoptosis, suggesting that they are not secondary in nature, further supported by the fact that acute disruption of c-myc recapitulates chromatin defects (Knoepfler et al. 2002; Knoepfler et al. 2006). At the molecular level, loss of c-myc results in reduction of histone $\mathrm{H} 3$ and $\mathrm{H} 4$ acetylation but a marked increase in trimethylation of histone H3 lysine 9, consistent with an increase in heterochromatin in these cells. These observations strongly suggest that $\mathrm{c}-\mathrm{myc}$ is a regulator of global chromatin structure and contributes to maintaining euchromatin in an open, accessible state (Knoepfler et al. 2006; reviewed in Knoepfler 2007).

The mechanism by which c-myc functions in higher-order chromatin organization is unclear. One model suggests that that the protein attracts histone modifying activities and chromatin remodeling machinery to its many binding sites spread throughout the genome, thus, exposing large portions of the genome to them (reviewed in Knoepfler 2007). Alternatively, the global effect of c-myc could be mediated by misregulation of a single, or a few, global chromatin modifiers. A candidate for this model is the histone acetyltransferase GCN5, which is a known target gene of c-myc (Knoepfler et al. 2006). Its up-regulation on loss of c-myc may contribute to some of the observed changes in histone modification and possibly chromatin structure. The involvement of cmyc in higher-order chromatin structure, and particularly its effect upon histone acetylation, is of potential clinical relevance because c-myc is misregulated in a wide range of tumors and modulators of histone modifications are 
T. Misteli

increasingly explored as potential anticancer drugs.

\section{HP1}

Heterochromatin protein 1 (HP1) is a prominent structural component of heterochromatin (Fanti and Pimpinelli 2008). The family of HP1 proteins is evolutionarily conserved and in higher eukaryotes exists as three isoforms: $\mathrm{HP} 1 \alpha, \beta$, and $\gamma$. HP1 proteins bind specifically via their chromo-domain to trimethylated lysine 9 on histone H3, enriched in heterochromatin, but are also present interdispersed throughout the genome (reviewed in Fanti and Pimpinelli 2008). The protein is thought to crosslink multiple chromatin fibers by multimerization via its chromo-shadow domain thus leading to chromatin compaction. In addition to its structural role in heterochromatin, HP1 proteins can have both activating as well as repressive functions in gene expression. As silencing factors they are thought to condense chromatin in regulatory regions of target genes, particularly at promoters, thus preventing efficient transcription initiation. As activators, they might similarly create a particular chromatin environment conducive to transcription (reviewed in Fanti and Pimpinelli 2008).

HP1 has been implicated in cancer in several ways. Reduced expression of various HP1 isoforms has been reported in multiple cancers including breast, brain, colon, and ovarian cancer (Dialynas et al. 2008). HP1 appears to be particularly important for cancer progression and the degree of HP1 loss correlates with poor prognosis, whereas increased HP1 levels correlate with reduced metastasis (Pomeroy et al. 2002; Ruginis et al. 2006). A direct causal effect of HP1 has been shown in breast cancer where experimental depletion of $\mathrm{HP} 1 \alpha$ from poorly invasive cancer cells increased their invasion capacity, but expression of $\mathrm{HP} 1 \alpha$ in highly invasive cells decreased their ability to do so (Kirschmann et al. 2000; Norwood et al. 2006). These observations suggest that HP1 $\alpha$ is a metastasis suppressor. The mechanism by which HP1 mediates this effect is not fully elucidated, but appears to involve global genome misregulation because a large number of genes with known connections to cancer and metastasis are affected by its depletion (Dialynas et al. 2008).

\section{HIGHER-ORDER CHROMATIN STRUCTURE IN DNA REPAIR AND TRANSLOCATIONS}

The first line of cellular defense against genome damage is the DNA repair machinery, which recognizes DNA lesions and corrects them before lasting damage can be done. There is increasing evidence that higher-order chromatin structure plays a key role in the efficiency of DNA repair (Goodarzi et al. 2009; reviewed in Misteli and Soutoglou 2009). For one, chromatin remodeling events are a prominent part of the DNA repair cascade (reviewed in Downs et al. 2007; Misteli and Soutoglou 2009). Early on, after a double-strand break (DSB) occurs, the chromatin around the break site rapidly decondenses, presumably to facilitate access of the repair machinery to the break site. Later in the repair process, several ATP-dependent chromatin-remodeling machines, mostly of the SWI/SNF family, are recruited to the break to prepare the chromatin for resection and ligation (reviewed in Downs et al. 2007; Misteli and Soutoglou 2009). The requirement for chromatin remodeling machines in efficient repair implies that the structural properties of chromatin influence the progression of the repair process and possibly also affect the susceptibility of chromatin to insult (Fig. 1). Direct evidence for this comes from the finding that irradiation-induced DSBs located in heterochromatin are more slowly repaired than the same breaks in euchromatin (Goodarzi et al. 2008). Although no kinetic studies have been reported to date, the most likely reason for the delay is the inefficient recruitment of the repair machinery into highly compacted chromatin because of reduced accessibility of binding sites. However, there is also the possibility that DSBs in euchromatin and heterochromatin use different repair mechanisms altogether. In support of this scenario, the repair of DSB in heterochromatin is significantly more sensitive to the activity of the major DNA repair kinase ATM 

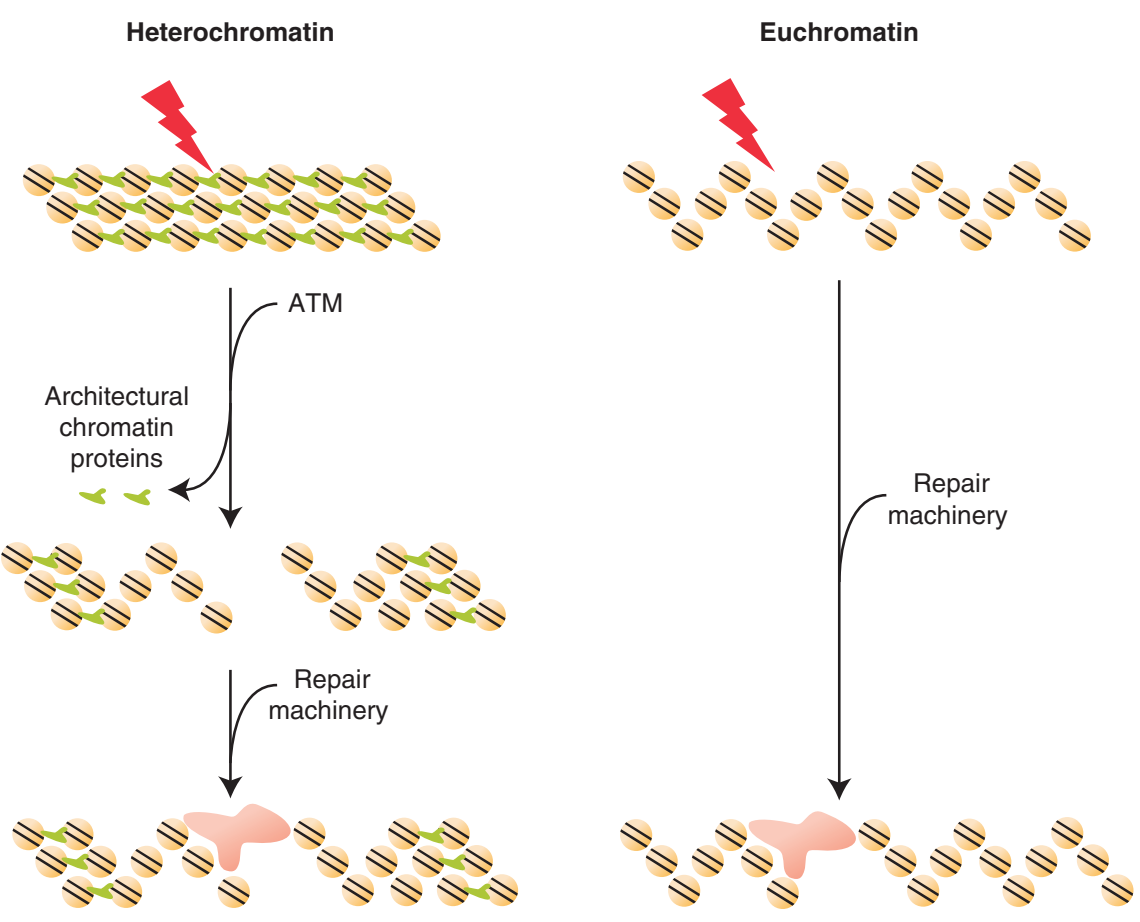

Figure 1. Higher-order chromatin structure and DNA repair. $(A)$ The condensation status of chromatin affects DNA repair. If a double strand break occurs in more densely packed heterochromatin region, architectural proteins (green) such as HP1, linker histone H1, or HMG proteins, associated with these domains, prevent access of the DNA repair machinery (red) and must be removed, possibly via action of the ATM kinase. Upon removal, the DNA repair machinery can gain more immediate access to the DSBs. $(B)$ In the less densely packed euchromatin regions, the repair machinery has freer access to the DSBs.

(Ataxia telangiectasia mutated) and slowly repaired, persistent DSBs are preferentially found within heterochromatin rather than in euchromatin (Goodarzi et al. 2009). Removal of several architectural chromatin proteins, including HP1, KAP-1, or HDAC1/2, alleviates the delay in ATM-mediated repair, suggesting that these heterochromatin components act as a barrier for efficient repair (Goodarzi et al. 2009) (Fig. 1). In line with this interpretation, HP1 is actively released from sites of DNA damage via specific phosphorylation of Thr51 and is only recruited at later time points, presumable when all critical repair factors have gained access to the DSB and normal chromatin structure needs to be re-established again (Ayoub et al. 2008; Luijsterburg et al. 2009). Further evidence that chromatin architecture affects the DNA response comes from observations on the HMGN1 protein (Highmobility group protein 1). HMGN1 is a sequence-independent global DNA binding protein implicated in transcriptional control via regulation of histone modifications (Hock et al. 2007). Mice lacking HMGN1 have a defective DNA damage response including reduced activating phosphorylation of ATM and its downstream targets CHK1 and CHK2, two key cell-cycle checkpoint signaling kinases (Birger et al. 2003; Kim et al. 2009). HMGN1 is not preferentially recruited to DSB but still plays an important role there. Loss of the protein leads to precocious accumulation of ATM on chromatin even in the absence of a DSB (Birger et al. 2003; Kim et al. 2009). The association with ATM on chromatin is controlled by the global level of acetylation of histone $\mathrm{H} 3$ on K14, which is known to be affected by HMGN1 (Lim et al. 
2005). Further evidence for a critical role of chromatin structure in susceptibility to DNA damage and repair comes from analysis of the DNA damage response in embryonic stem cells, which are unique in that they have a strict requirement to maintain their genomes in pristine conditions because any genomic defect will be propagated as the stem cells differentiate into various tissues. ES cells lacking the linker histone H1, one of the key global architectural components of chromatin, are characterized by less compacted chromatin and, remarkably, have a heightened DNA damage response and are able to repair DSB more rapidly after irradiation (Fan et al. 2005; Murga et al. 2007). This is likely because of the global decondensation of chromatin in these cells since the effect can be mimicked by treatment of cells with the histone-deacetylase inhibitor trichostatin $\mathrm{A}$, which also leads to chromatin decondensation (Murga et al. 2007). These observations suggest that chromatin structure directly affects susceptibility of the genome to damage and that different regions, such as heterochromatin and euchromatin, in the same genome respond differently to DNA damage. Defects in components involved in maintenance of higher-order chromatin structure are therefore candidates to promote mutations and therefore disease.

Higher-order chromatin structure may not only be important for global susceptibility of the genome to DNA damage, but may also contribute to the formation of specific chromosomal translocations. This may occur by local changes in chromatin structure, which create local fragile sites making them highly susceptible to breakage. An intriguing observation in support of this scenario has been provided by analysis of the molecular mechanisms involved in formation of a chromosomal translocation involved in ALCL (anaplastic large cell lymphoma) (Mathas et al. 2009). Many ALCL patients carry a translocation between chromosomes 2 and $5(\mathrm{t}(2 ; 5)(\mathrm{p} 23 ; \mathrm{q} 35))$ leading to the synthesis of a fusion protein between nucleophosmin and ALK (anaplastic lymphoma kinase), constitutively activating the kinase. Intriguingly, a significant fraction of ALCL patients with indistinguishable disease symptoms lack the $t(2 ; 5)$ translocation, clearly indicating the fusion protein is not necessary nor causal for disease. In analyzing the genes near the break points on chromosomes 2 and 5 , it was found that several genes adjacent to nucleophosmin and ALK were already highly expressed even in patients without the translocation (Mathas et al. 2009). Although not experimentally tested, these results suggest that the chromatin structure near the breakpoints is altered even in the absence of the translocation. Interestingly, patient cells lacking the $t(2 ; 5)$ translocation were more highly susceptible to form the $\mathrm{t}(2 ; 5)$ translocation than control cells, suggesting that the transcriptional activation of the flanking genes, and presumably the altered chromatin structure in these regions, predisposed cells to formation of the translocation (Mathas et al. 2009). It is attractive to speculate, that the transcriptional activation of the break-point proximal regions were brought about by improper recruitment of transcription factors to these sites, activating them and altering the local chromatin increasing their susceptibility to breakage and translocation. In this model, the first step in formation of a chromosomal translocation is the aberrant activation of genome regions, leading to their decondensation. Direct support for this scenario comes from recent finding that translocation breakpoints in prostate cancer contain binding sites for androgen receptor (AR), a transcriptional activator. Upon treatment with androgens, AR binds to these sites leading to chromatin remodeling, which may increase the susceptibility to DNA damage (Lin et al. 2009). This model is also supported by the finding that active alleles, but not inactive ones, of the translocation prone MYC and IGH loci, whose translocation causes Burkitts lymphoma, often associate with each other in the threedimensional (3D) space of the nucleus, predisposing them for translocation (Roix et al. 2003; Osborne et al. 2007). It will be important to further test this model by determining whether other translocation breakpoints are also characterized by heightened transcription and what the molecular mechanisms in this cascade of events are. 


\section{SPATIAL CHROMOSOME ORGANIZATION IN CANCER}

Chromosomal abnormalities in the form of translocations and aneuploidy are a general hallmark of cancer cells. While aneuploidy is generated by unequal chromosome segregation during mitosis, translocations occur during interphase by illegitimate joining of two or multiple chromosomes containing persistently damaged DNA such as double-strand breaks (DSBs). Because the formation of a translocation requires the physical interaction of the involved chromosomes, the spatial arrangement of chromosomes, i.e. their localization relative to each other, in the nucleus may contribute to determining which chromosomes undergo translocations (reviewed in Misteli
2004; Meaburn et al. 2007). It has been proposed that translocations preferentially occur between neighboring chromosomes but not between more distantly located chromosomes (Fig. 2). This is a particularly intriguing, and important, possibility because it is now well established that genomes are nonrandomly distributed within the nuclear space in a cell-type and tissue-specific manner (reviewed in Cremer and Cremer 2001; Misteli 2007). A body of correlative evidence indeed suggests that the nonrandomness in spatial genome organization contributes to determining translocation partners (reviewed in Meaburn et al. 2007).

A first hint for a role of spatial genome organization in the formation of translocations comes from the finding that in humans the acrocentric chromosomes, which contain nucleolar

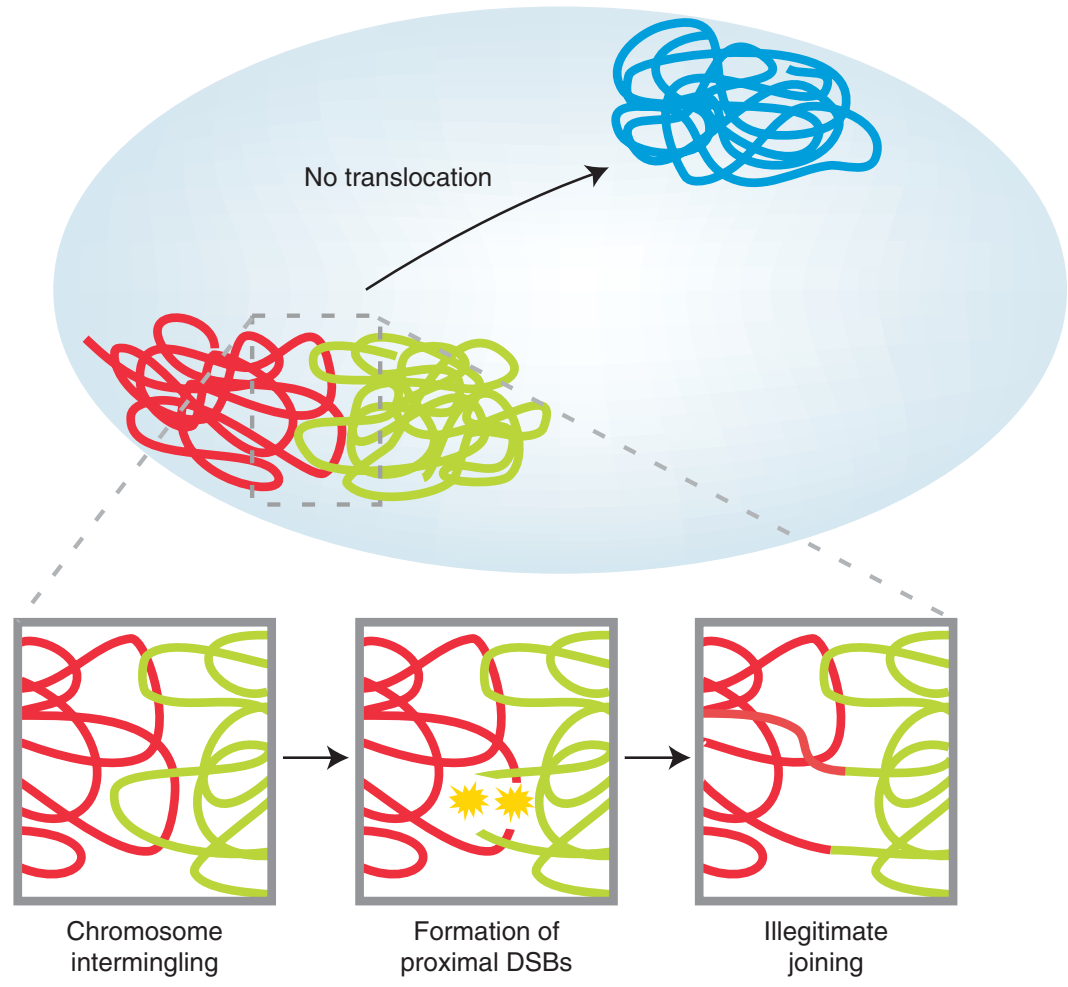

Figure 2. Spatial organization of chromosomes in the formation of cancer translocations. Translocations preferentially occur between proximally positioned chromosomes (red, green), and only rarely between distally located chromosomes (blue). Closely juxtaposed double-strand breaks (yellow stars) occurring at the interface between chromosomes create free chromosome ends, which may recombine to form a chromosome translocation by illegitimate joining. 


\section{T. Misteli}

organizing regions (NORs) and cluster in nucleoli, undergo translocations to form Robertsonian translocations with higher frequency than many other chromosomes (Therman et al. 1989). This is presumably because of their tight packing in the nucleolus, increasing their probability of illegitimate joining any time two or more DSBs are not promptly repaired. Similarly, the translocation frequency of chromosomes lining the nuclear envelope is elevated compared with internally located chromosomes (Bickmore and Teague 2002). In addition to these general observations, many examples of correlations between physical proximity and translocation frequencies of specific chromosomes exist (reviewed in Meaburn et al. 2007). Mouse chromosomes MMU12, 14, and 15 are frequently involved in translocations in mouse lymphoma and these chromosomes are found in pairs or in triplet clusters in more than $60 \%$ of normal lymphocytes before the formation of a translocation, placing them in close spatial proximity and predisposing them to undergo translocations among each other once persistent DSBs occur (Parada et al. 2002). Furthermore, chromosomes MMU5 and MMU6, which are frequently involved in translocations in kidney, are neighbors in more than $40 \%$ of normal hepatocytes (Parada et al. 2004) and human chromosomes HSA12 and 16, frequently translocated in adipocyte cancers, are in close proximity in differentiated adipocytes (Kuroda et al. 2004).

Translocations often occur between specific regions or genes of the two involved chromosomes and studies on the localization of the involved chromosomal breakpoints further strengthen the case for a role of spatial proximity in determining translocation frequency. The $B C R$ and $A B L$ genes, located on chromosomes 9 and 22, whose translocation leads to formation of a fusion protein, which is causal in chronic myeloid leukemia, are found in proximity to each other in normal hematopoietic cells at much higher frequency than would be expected based on their random distribution and significantly higher than observed for various other gene loci in the same nuclei (Lukasova et al. 1997; Neves et al. 1999; Bartova et al. 2000).
In B-cells, the PML and RAR $\alpha$ genes, located on chromosomes 15 and 17, respectively, which translocate to cause promyelocytic leukemia, are in close proximity (Neves et al. 1999) and the MYC gene on chromosome 8 and the $I G H$ locus on chromosome 14, whose translocation leads to Burkitts lymphoma, are frequently juxtaposed (Roix et al. 2003; Osborne et al. 2007). Importantly, two less frequent Burkitts lymphoma translocation partners, Igא on chromosome 11, and $I g \lambda$ on chromosome 22, show less frequent association with their translocation partner MYC correlating with their limited propensity to translocate with MYC (Roix et al. 2003). Proximity effects on translocation formation are not limited to gene loci located on different chromosomes but also apply to events on the same chromosome. The RET and the H4 genes are frequent translocation partners in thyroid tumors and are both located on chromosome 10 about $30 \mathrm{MB}$ apart but are brought together in 3D space by looping of the chromosome fiber, thus juxtapositioning, and predisposing, them to undergo a mutual translocation resulting in an internal chromosome reversion (Nikiforova et al. 2000). In addition to these correlative observations, direct evidence for a role of proximity comes from irradiation experiments in cells from anaplastic large cell lymphoma (ALCL). When cells which contain nonrandom pairs of closely positioned chromosome 2 and 5 were irradiation to induce DSBs, these two chromosomes formed translocations with significantly higher frequency than in cells where they were distant from each other, suggesting that their proximity facilitated their translocation (Mathas et al. 2009).

A role for proximity in determining translocation outcome is in line with the wellestablished fact that translocation frequencies differ among tissues (Mitelman et al. 2007). For example, although translocations between mouse chromosomes 12 and 14 are often associated with lymphoma, translocations between chromosomes 5 and 6 are most prominently found in cancers of the kidney. The tissue-specificity of these translocations is paralleled by tissue specific organization of the involved chromosomes whereby chromosomes 12 and 14 pair in 
lymphocytes but not in hepatocyes, but chromosomes 5 and 6 pair in hepatocytes and not lymphocytes. It seems that the preference in translocation frequency is thus a reflection of the tissue-specific arrangement of the involved translocation partners (Parada et al. 2004).

The conclusion that the relative position of translocating regions is important in determining their interaction relies on the assumption that once DSBs form in a chromosome, the generated free ends are immobile and cannot widely roam the nuclear space to find other broken chromosome ends. Several lines of evidence indeed suggest that broken chromosome ends are immobile in the nucleus. Visualization of DSBs in chemically fixed and living cells, show that irradiated genome regions remain in place for extended periods of time (Nelms et al. 1998; Kruhlak et al. 2006). Furthermore, single fluorescently tagged DSBs induced by sitespecific cutting with a restriction endonuclease remained stationary within the nuclear space (Soutoglou et al. 2007). These experiments also revealed that the radius of motion of a DSB is limited to about $250 \mathrm{~nm}$. The mechanisms responsible for DSB immobility are unknown but might involve tethering of DSBs to DNA repair foci.

The picture emerging from these observations is that translocations occur preferentially between already closely positioned genome regions and do not involve an extensive search process during which DSBs on multiple chromosomes diffuse through the nucleus in search of other DSBs (Fig. 2). Translocations will only take place if two DSBs occur in chromatin of two adjacent, and likely intermingling, chromosomes and are not separated by more than $\sim 500 \mathrm{~nm}$, corresponding to the combined radius of motion of the two DSBs (Fig. 2). This model is in line with the finding that translocation frequencies between chromosomes not only correlate with their relative position but with the degree of overlap between the chromatin of adjacent chromosomes (Branco and Pombo 2006).

The genome is continuously exposed to external insults such as irradiation and chemicals, which lead to DNA damage and DSBs.
The immobility of DSBs likely acts as a protective mechanism to ensure genome stability and it explains the relatively low frequency by which translocations occur. It is estimated that the genome in an individual cell suffers several thousand DNA damage events daily, including DSBs, however, translocations occur at frequencies of $\sim 10^{-7}$. If DSBs were highly mobile within the nucleus, translocation frequencies would be much higher because randomly occurring DSBs would be able to rapidly find other DSBs in the same genome to readily undergo translocations. The intrinsic immobility of DSBs in the nucleus prevents the juxtaposition of multiple broken chromosome ends and allows the DNA repair machinery to resolve the defect before a translocation partner can be found. In this sense, the higher-order organization of the chromosomes and the chromatin fiber, and their immobility, act as a guardian of the genome.

\section{HIGHER-ORDER CHROMATIN STRUCTURE IN AGING}

Aging is characterized by several global changes in chromatin structure and function. Structurally, in aged cells several key architectural chromatin proteins, including the structural heterochromatin protein HP1, are lost because of posttranslational degradation (Scaffidi and Misteli 2006; Pegoraro et al. 2009). Similarly, in the premature aging disorder HutchinsonGilford Progeria Syndrome (HGPS), caused by a mutation in lamin $\mathrm{A}$, one of the major architectural elements of the mammalian nucleus, the same chromatin proteins are lost and, most likely as a consequence of their absence, morphologically discernible heterochromatin blocks and peripheral heterochromatin are largely missing from HGPS patient cells (Goldman et al. 2004; Scaffidi and Misteli 2006). These defects are because of downregulation of the NUcleosome Remodeling and Deacetylase (NURD) chromatin remodeling complex (Pegoraro et al. 2009). Several components of NURD, including its catalytic histone deacetylase I (HDAC 1), are lost in normally aged and HGPS patient cells and removal 
of this complex from normal cells is sufficient to recapitulate most major aging-associated chromatin defects (Pegoraro et al. 2009). These observations are supported by the finding of reduced activity of several histone deacetylases in aged mice (Shen et al. 2008). Aging-related structural defects in chromatin are also seen in yeast $S$. cerevisiae where instability of ribosomal repeats in the nucleolus have been associated with aging (Sinclair and Guarente 1997) and in Caenorhabditis elegans where both internal and peripheral heterochromatin blocks are lost during aging (Haithcock et al. 2005).

The aging-associated defects in chromatin structure have various functional consequences. To start with, aged genomes are characterized by increased DNA damage and high levels of persistent DNA breaks, possibly brought about by structural changes, which increase the susceptibility of the genome to damage. Furthermore, probably as a consequence of loss of pericentromeric heterochromatin structure, physiologically aged and premature aged cells express normally silenced heterochromatic satellite III repeats (Gaubatz and Cutler 1990; Shumaker et al. 2006). Physiologically aged and prematurely aged genomes also undergo wide-ranging changes in epigenetic modifications. Global DNA methylation is reduced in many aged mammalian tissues (Wilson and Jones 1983; Fraga and Esteller 2007) and several histone modifications are affected during aging. In particularly, and in line with the morphological changes in chromatin, the heterochromatinassociated trimethylation of histone $\mathrm{H} 3$ lysine 9 and the transcriptionally repressive trimethylation of histone H3 lysine 27 are largely lost in aged and prematurely aged cells (Scaffidi and Misteli 2006; Shumaker et al. 2006). Conversely, global trimethylation of $\mathrm{H} 4$ lysine 20 increases with age (Sarg et al. 2002). In S. cerevisiae, acetylation of $\mathrm{H} 4$ lysine 16 increases, and that of $\mathrm{H} 3$ lysine 56 decreases with age, whereas several other modifications are unchanged (Dang et al. 2009). The level of these acetylation events is dependent on the histone deacetylase Sir2 and is counteracted by the acetyltransferase Sas2 (Dang et al. 2009). Evidence that these changes in histone modifications are not merely bystanders in the aging process but actively drive it comes from the finding that mutations in histones $\mathrm{H} 3$ and $\mathrm{H} 4$, which interfere with their acetylation on lysine 16 or lysine 56 prolong lifespan (Dang et al. 2009). Given these extensive changes in histone modifications, not surprisingly, aged cells show dramatic and global misregulation of gene expression. Although some of these changes are likely part of specific aging-related gene expression programs including inflammation and cellular stress responses, others likely occur largely stochastically because of random changes in epigenetic modifications and chromatin structure.

The mechanisms that drive chromatin and epigenetic changes during aging are currently unknown (Fig. 3). It has been suggested that the epigenetic alterations are largely triggered by DNA damage (reviewed in Oberdoerffer and Sinclair 2007). In this scenario, randomly occurring DNA damage leads to chromatin remodeling and to redistribution of chromatin modifiers within the genome with modifiers being recruited away from their normal sites so that they can engage in the repair of the DNA damage and others being recruited to newly available sites. The redistribution of histone modifiers then leads to activation of aging-related gene expression programs and contribute to general, random misregulation of genes globally (Fig. 3) (reviewed in Oberdoerffer and Sinclair 2007). This model is supported by the observed redistribution of the histone deacetylase SIRT1, which has previously been implicated in aging, in response to DNA damage and its association with genes, which are deregulated during aging (Oberdoerffer et al. 2008). However, the model does not fully account for the observation that several epigenetic modifiers are lost, rather than redistributed within the genome, in normally and prematurely aged cells (Shen et al. 2008; Pegoraro et al. 2009). Furthermore, at least in the premature aging syndrome HGPS, DNA damage is a late event and is unlikely the trigger for the observed epigenetic changes because the loss of key chromatin proteins, such as NURD, occurs before DNA damage and is followed by changes in chromatin structure and epigenetic 
Genome Organization in Disease

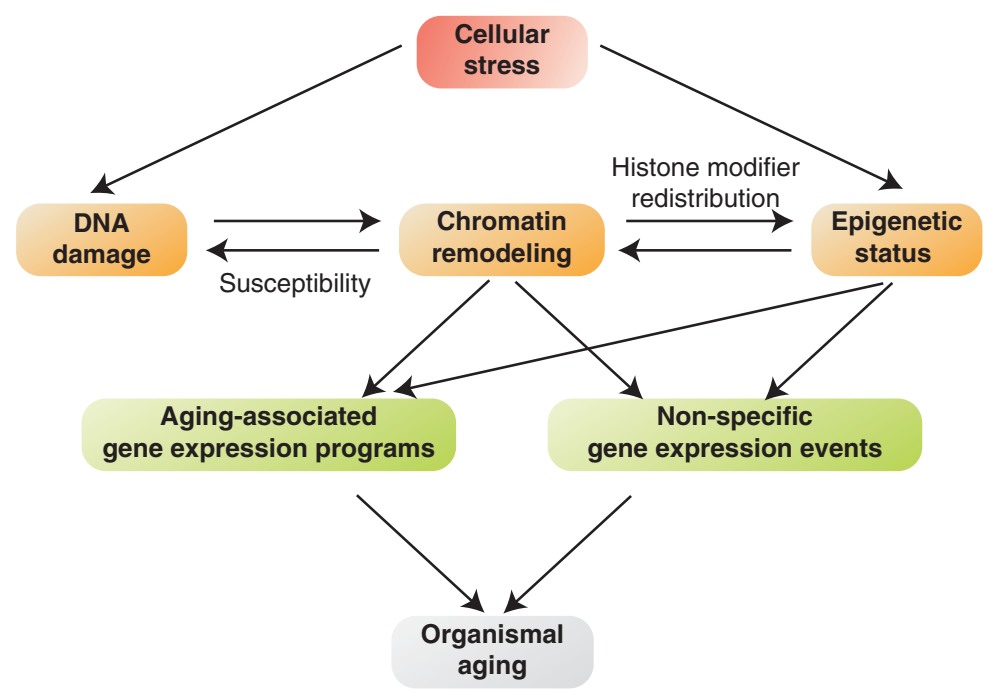

Figure 3. Chromatin effects in aging. A complex network of interactions links chromatin structure to aging. Cellular stress may directly induce changes in the epigenetic status of the genome leading to local and global chromatin remodeling, which in turn may make the genome more susceptible to DNA damage. Cellular stress may also cause DNA lesions itself. As part of the cellular response to these lesions, chromatin remodeling events occur and may lead to redistribution of epigenetic modifiers away from their regular binding sites and toward inappropriate targets, thus altering the epigenetic state of the genome. Alterations in global chromatin structure and epigenetic status lead to activation of gene expression programs including specific-aging associated programs such as activation of inflammation and cellular stress responses, but they likely also contribute to random misregulation of genes throughout the genome. These specific and nonspecific misregulation events likely act in a feed-back loop to further destabilize the epigenetic homeostasis of the aging genome.

modifications and only eventually the appearance of DNA damage (Pegoraro et al. 2009). An alternative model is that aging is primarily driven by steady loss of epigenetic modifiers brought about by continuous, often low level, exposure to cellular stressors (Fig. 3). The loss or aberrant function of epigenetic modifiers alters the makeup of the global epigenome and as a consequence global chromatin structure, including loss of heterochromatin regions. This loss of heterochromatin may make the genome more susceptible to DNA damage, which, in a feedback mechanism, triggers further global epigenetic changes including aging-associated changes in gene expression programs and random misregulation of genes globally (Fig. 3). These considerations make it clear that although the involvement of epigenetic modifications, chromatin structure and DNA damage are now clearly established as important components of the aging process, the precise interdependencies of these events remain to be elucidated (Fig. 3).

\section{CONCLUSIONS}

The organization of the genome into higherorder chromatin and chromosomes is one of the fundamental features of eukaryotic cells. Not surprisingly, proper organization of the genome is essential for faithful genome function and defects lead to disease. Structural chromatin defects may come about directly by mutations or misexpression of bona-fide architectural chromatin proteins or chromatin remodeling activities. Alternatively, chromatin structure may be compromised because of impaired function of histone modifying activities, which in turn lead to structural chromatin changes. The number of known diseases 
involving structural abnormalities of chromatin is likely to grow rapidly in the next few years. In many diseases involving gene misregulation, often assumed to be because of altered epigenetic modifications, chromatin structure has not been analyzed and it is possible that many "epigenetic" diseases are in reality diseases of chromatin structure. Furthermore, genome wide mapping of single-nucleotide polymorphisms is likely to reveal a significant number of novel mutations, which affect higher-order chromatin structure. In addition to chromatin structural defects leading to specific diseases, the importance of global higher-order chromatin organization as a protective means in the maintenance of genome integrity as a shield against the continued onslaught of DNA damaging agents is rapidly becoming clear. This function is emerging as an integral mechanism in pathological processes such as formation of cancer translocations and aging. Elucidation of the precise role and molecular mechanisms involved in maintaining chromatin structure and its role will be essential for our understanding of these all-important events.

The involvement of higher-order chromatin structure, and nuclear architecture in general, in disease is a powerful example of how fundamental cellular processes are relevant for disease. We often think of the study of basic cellular function as a separate discipline from clinical research. However, we are now seeing a dramatic convergence of basic and disease research. As we are making progress in outlining at the molecular level the mechanisms underlying cellular function, links to, often unexpected, diseases are being discovered. On the other hand, the genomic tools, which have led to a dramatic increase in the rate of discovery of disease genes highlight the relevance of basic cellular machinery for disease because for each identified disease gene, its basic mechanisms of action must be elucidated to truly understand the disease. The combination of increasingly detailed insight into basic biological processes and the accelerated rate of disease gene discovery provides a powerful, and promising, forum for the development of novel diagnostic and therapeutic strategies in the future.

\section{REFERENCES}

Ayoub N, Jeyasekharan AD, Bernal JA, Venkitaraman AR. 2008. HP1- $\beta$ mobilization promotes chromatin changes that initiate the DNA damage response. Nature 453: 682-686.

Bartova E, Kozubek S, Kozubek M, Jirsova P, Lukasova E, Skalnikova M, Buchnickova K. 2000. The influence of the cell cycle, differentiation and irradiation on the nuclear location of the abl, bcr and c-myc genes in human leukemic cells. Leuk Res 24: 233-241.

Belmont AS. 2006. Mitotic chromosome structure and condensation. Curr Opin Cell Biol 18: 632-638.

Bickmore WA, Teague P. 2002. Influences of chromosome size, gene density and nuclear position on the frequency of constitutional translocations in the human population. Chromosome Res 10: 707-715.

Birger Y, West KL, Postnikov YV, Lim JH, Furusawa T, Wagner JP, Laufer CS, Kraemer KH, Bustin M. 2003. Chromosomal protein HMGN1 enhances the rate of DNA repair in chromatin. EMBO J 22: 1665-1675.

Branco MR, Pombo A. 2006. Intermingling of chromosome territories in interphase suggests role in translocations and transcription-dependent associations. PLoS Biol 4: e138.

Cai S, Han HJ, Kohwi-Shigematsu T. 2003. Tissue-specific nuclear architecture and gene expression regulated by SATB1. Nat Genet 34: 42-51.

Cai S, Lee CC, Kohwi-Shigematsu T. 2006. SATB1 packages densely looped, transcriptionally active chromatin for coordinated expression of cytokine genes. Nat Genet 38: $1278-1288$.

Cremer T, Cremer C. 2001. Chromosome territories, nuclear architecture and gene regulation in mammalian cells. Nat Rev Genet 2: 292-301.

Cremer T, Cremer M, Dietzel S, Muller S, Solovei I, Fakan S. 2006. Chromosome territories - a functional nuclear landscape. Curr Opin Cell Biol 18: 307-316.

D’Ambrosio C, Schmidt CK, Katou Y, Kelly G, Itoh T, Shirahige K, Uhlmann F. 2008. Identification of cis-acting sites for condensin loading onto budding yeast chromosomes. Genes Dev 22: 2215-2227.

Dang W, Steffen KK, Perry R, Dorsey JA, Johnson FB, Shilatifard A, Kaeberlein M, Kennedy BK, Berger SL. 2009. Histone H4 lysine 16 acetylation regulates cellular lifespan. Nature 459: 802-807.

Dialynas GK, Vitalini MW, Wallrath LL. 2008. Linking Heterochromatin Protein 1 (HP1) to cancer progression. Mutat Res 647: 13-20.

Dickinson LA, Joh T, Kohwi Y, Kohwi-Shigematsu T. 1992. A tissue-specific MAR/SAR DNA-binding protein with unusual binding site recognition. Cell 70: 631-645.

Dion V, Wilson JH. 2009. Instability and chromatin structure of expanded trinucleotide repeats. Trends Genet (in press).

Donze D, Adams CR, Rine J, Kamakaka RT. 1999. The boundaries of the silenced HMR domain in Saccharomyces cerevisiae. Genes Dev 13: 698-708.

Dorsett D. 2009. Cohesin, gene expression and development: Lessons from Drosophila. Chromosome Res 17: $185-200$. 
Dorsett D, Krantz ID. 2009. On the molecular etiology of Cornelia de Lange syndrome. Ann N Y Acad Sci 1151: $22-37$.

Downs JA, Nussenzweig MC, Nussenzweig A. 2007. Chromatin dynamics and the preservation of genetic information. Nature 447: 951-958.

Eggermann T, Eggermann K, Schonherr N. 2008. Growth retardation versus overgrowth: Silver-Russell syndrome is genetically opposite to Beckwith-Wiedemann syndrome. Trends Genet 24: 195-204.

Eilers M, Eisenman RN. 2008. Myc's broad reach. Genes Dev 22: $2755-2766$.

Fan Y, Nikitina T, Zhao J, Fleury TJ, Bhattacharyya R, Bouhassira EE, Stein A, Woodcock CL, Skoultchi AI. 2005. Histone $\mathrm{H} 1$ depletion in mammals alters global chromatin structure but causes specific changes in gene regulation. Cell 123: 1199-1212.

Fanti L, Pimpinelli S. 2008. HP1: A functionally multifaceted protein. Curr Opin Genet Dev 18: 169-174.

Felsenfeld G, Groudine M. 2003. Controlling the double helix. Nature 421: 448-453.

Fraga MF, Esteller M. 2007. Epigenetics and aging: The targets and the marks. Trends Genet 23: 413-418.

Fraser P, Bickmore W. 2007. Nuclear organization of the genome and the potential for gene regulation. Nature 447: 413-417.

Gaubatz JW, Cutler RG. 1990. Mouse satellite DNA is transcribed in senescent cardiac muscle. J Biol Chem 265: 17753-17758.

Glynn EF, Megee PC, Yu HG, Mistrot C, Unal E, Koshland DE, DeRisi JL, Gerton JL. 2004. Genome-wide mapping of the cohesin complex in the yeast Saccharomyces cerevisiae. PLoS Biol 2: E259.

Goldman RD, Shumaker DK, Erdos MR, Eriksson M, Goldman AE, Gordon LB, Gruenbaum Y, Khuon S, Mendez M, Varga R, et al. 2004. Accumulation of mutant lamin A causes progressive changes in nuclear architecture in Hutchinson-Gilford progeria syndrome. Proc Natl Acad Sci 101: 8963-8968.

Goodarzi AA, Noon AT, Jeggo PA. 2009. The impact of heterochromatin on DSB repair. Biochem Soc Trans 37: 569-576.

Goodarzi AA, Noon AT, Deckbar D, Ziv Y, Shiloh Y, Lobrich M, Jeggo PA. 2008. ATM signaling facilitates repair of DNA double-strand breaks associated with heterochromatin. Mol Cell 31: 167-177.

Haithcock E, Dayani Y, Neufeld E, Zahand AJ, Feinstein N, Mattout A, Gruenbaum Y, Liu J. 2005. Age-related changes of nuclear architecture in Caenorhabditis elegans. Proc Natl Acad Sci 102: 16690-16695.

Han HJ, Russo J, Kohwi Y, Kohwi-Shigematsu T. 2008. SATB1 reprogrammes gene expression to promote breast tumour growth and metastasis. Nature 452: 187-193.

Heintzman ND, Hon GC, Hawkins RD, Kheradpour P, Stark A, Harp LF, Ye Z, Lee LK, Stuart RK, Ching CW, et al. 2009. Histone modifications at human enhancers reflect global cell-type-specific gene expression. Nature 459: $108-112$.

Hock R, Furusawa T, Ueda T, Bustin M. 2007. HMG chromosomal proteins in development and disease. Trends Cell Biol 17: 72-79.
Kim YC, Gerlitz G, Furusawa T, Catez F, Nussenzweig A, Oh KS, Kraemer KH, Shiloh Y, Bustin M. 2009. Activation of ATM depends on chromatin interactions occurring before induction of DNA damage. Nat Cell Biol 11: 92-96.

Kirschmann DA, Lininger RA, Gardner LM, Seftor EA, Odero VA, Ainsztein AM, Earnshaw WC, Wallrath LL, Hendrix MJ. 2000. Down-regulation of HP1Hsalpha expression is associated with the metastatic phenotype in breast cancer. Cancer Res 60: 3359-3363.

Knoepfler PS. 2007. Myc goes global: New tricks for an old oncogene. Cancer Res 67: 5061-5063.

Knoepfler PS, Cheng PF, Eisenman RN. 2002. N-myc is essential during neurogenesis for the rapid expansion of progenitor cell populations and the inhibition of neuronal differentiation. Genes Dev 16: 2699-2712.

Knoepfler PS, Zhang XY, Cheng PF, Gafken PR, McMahon SB, Eisenman RN. 2006. Myc influences global chromatin structure. EMBO J 25: 2723-2734.

Krantz ID, McCallum J, DeScipio C, Kaur M, Gillis LA, Yaeger D, Jukofsky L, Wasserman N, Bottani A, Morris CA, et al. 2004. Cornelia de Lange syndrome is caused by mutations in NIPBL, the human homolog of Drosophila melanogaster Nipped-B. Nat Genet 36: 631-635.

Kruhlak MJ, Celeste A, Dellaire G, Fernandez-Capetillo O, Muller WG, McNally JG, Bazett-Jones DP, Nussenzweig A. 2006. Changes in chromatin structure and mobility in living cells at sites of DNA double-strand breaks. $J$ Cell Biol 172: 823-834.

Kuroda M, Tanabe H, Yoshida K, Oikawa K, Saito A, Kiyuna T, Mizusawa H, Mukai K. 2004. Alteration of chromosome positioning during adipocyte differentiation. $J$ Cell Sci 117: 5897-5903.

Kurukuti S, Tiwari VK, Tavoosidana G, Pugacheva E, Murrell A, Zhao Z, Lobanenkov V, Reik W, Ohlsson R. 2006. CTCF binding at the H19 imprinting control region mediates maternally inherited higher-order chromatin conformation to restrict enhancer access to Igf2. Proc Natl Acad Sci 103: 10684-10689.

Lanctot C, Cheutin T, Cremer M, Cavalli G, Cremer T. 2007. Dynamic genome architecture in the nuclear space: Regulation of gene expression in three dimensions. Nat Rev Genet 8: 104-115.

Lengronne A, Katou Y, Mori S, Yokobayashi S, Kelly GP, Itoh T, Watanabe Y, Shirahige K, Uhlmann F. 2004. Cohesin relocation from sites of chromosomal loading to places of convergent transcription. Nature 430: 573-578.

Li T, Hu JF, Qiu X, Ling J, Chen H, Wang S, Hou A, Vu TH, Hoffman AR. 2008. CTCF regulates allelic expression of Igf2 by orchestrating a promoter-polycomb repressive complex 2 intrachromosomal loop. Mol Cell Biol 28: 6473-6482.

Libby RT, Hagerman KA, Pineda VV, Lau R, Cho DH, Baccam SL, Axford MM, Cleary JD, Moore JM, Sopher BL, et al. 2008. CTCF cis-regulates trinucleotide repeat instability in an epigenetic manner: A novel basis for mutational hot spot determination. PLoS Genet 4: e1000257.

Lim JH, West KL, Rubinstein Y, Bergel M, Postnikov YV, Bustin M. 2005. Chromosomal protein HMGN1 enhances the acetylation of lysine 14 in histone H3. EMBO J 24: 3038-3048. 


\section{T. Misteli}

Lin C, Yang L, Tanasa B, Hutt K, Ju B, Ohgi KA, Zhang J, Rose DW, Fu X-D, Glass CK, Rosenfeld MG. 2009. Nuclear receptor-induced chromosomal proximity and DNA breaks underlie specific translocations in cancer. Cell 139: 1069-1083

Ling JQ, Li T, Hu JF, Vu TH, Chen HL, Qiu XW, Cherry AM, Hoffman AR. 2006. CTCF mediates interchromosomal colocalization between Igf2/H19 and Wsb1/Nf1. Science 312: $269-272$.

Luijsterburg MS, Dinant C, Lans H, Stap J, Wiernasz E, Lagerwerf S, Warmerdam DO, Lindh M, Brink MC, Dobrucki JW, et al. 2009. Heterochromatin protein 1 is recruited to various types of DNA damage. $J$ Cell Biol 185: $577-586$.

Lukasova E, Kozubek S, Kozubek M, Kjeronska J, Ryznar L, Horakova J, Krahulcova E, Horneck G. 1997. Localisation and distance between $\mathrm{ABL}$ and BCR genes in interphase nuclei of bone marrow cells of control donors and patients with chronic myeloid leukaemia. Hum Genet 100: $525-535$.

Majumder P, Gomez JA, Chadwick BP, Boss JM. 2008. The insulator factor CTCF controls MHC class II gene expression and is required for the formation of long-distance chromatin interactions. J Exp Med 205: 785-798.

Mathas S, Kreher S, Meaburn KJ, Johrens K, Lamprecht B, Assaf C, Sterry W, Kadin ME, Daibata M, Joos S, et al. 2009. Gene deregulation and spatial genome reorganization near breakpoints prior to formation of translocations in anaplastic large cell lymphoma. Proc Natl Acad Sci 106: $5831-5836$.

McNairn AJ, Gerton JL. 2008. Cohesinopathies: One ring, many obligations. Mutat Res 647: 103-111.

Meaburn KJ, Misteli T. 2007. Cell biology: Chromosome territories. Nature 445: 379-781.

Meaburn KJ, Misteli T, Soutoglou E. 2007. Spatial genome organization in the formation of chromosomal translocations. Semin Cancer Biol 17: 80-90.

Misteli T. 2004. Spatial positioning: A new dimension in genome function. Cell 119: 153-156.

Misteli T. 2007. Beyond the sequence: Cellular organization of genome function. Cell 128: 787-800.

Misteli T, Soutoglou E. 2009. The emerging role of nuclear architecture in DNA repair and genome maintenance. Nat Rev Mol Cell Biol 10: 243-254.

Misulovin Z, Schwartz YB, Li XY, Kahn TG, Gause M, MacArthur S, Fay JC, Eisen MB, Pirrotta V, Biggin MD, Dorsett D. 2008. Association of cohesin and Nipped-B with transcriptionally active regions of the Drosophila melanogaster genome. Chromosoma 117: 89-102.

Mitelman F, Johansson B, Mertens F. 2007. The impact of translocations and gene fusions on cancer causation. Nat Rev Cancer 7: 233-245.

Murga M, Jaco I, Fan Y, Soria R, Martinez-Pastor B, Cuadrado M, Yang SM, Blasco MA, Skoultchi AI, FernandezCapetillo O. 2007. Global chromatin compaction limits the strength of the DNA damage response. J Cell Biol 178: $1101-1108$

Nelms BE, Maser RS, MacKay JF, Lagally MG, Petrini JH. 1998. In situ visualization of DNA double-strand break repair in human fibroblasts. Science 280: 590-592.
Neves H, Ramos C, da Silva MG, Parreira A, Parreira L. 1999. The nuclear topography of ABL, BCR, PML, and RARalpha genes: evidence for gene proximity in specific phases of the cell cycle and stages of hematopoietic differentiation. Blood 93: 1197-1207.

Nikiforova MN, Stringer JR, Blough R, Medvedovic M, Fagin JA, Nikiforov YE. 2000. Proximity of chromosomal loci that participate in radiation-induced rearrangements in human cells. Science 290: 138-141.

Norwood LE, Moss TJ, Margaryan NV, Cook SL, Wright L, Seftor EA, Hendrix MJ, Kirschmann DA, Wallrath LL. 2006. A requirement for dimerization of HP1Hsalpha in suppression of breast cancer invasion. J Biol Chem 281: $18668-18676$.

Oberdoerffer P, Sinclair DA. 2007. The role of nuclear architecture in genomic instability and ageing. Nat Rev Mol Cell Biol 8: 692-702.

Oberdoerffer P, Michan S, McVay M, Mostoslavsky R, Vann J, Park SK, Hartlerode A, Stegmuller J, Hafner A, Loerch P, et al. 2008. SIRT1 redistribution on chromatin promotes genomic stability but alters gene expression during aging. Cell 135: 907-918.

Orr HT, Zoghbi HY. 2007. Trinucleotide repeat disorders. Annu Rev Neurosci 30: 575-621.

Osborne CS, Chakalova L, Mitchell JA, Horton A, Wood AL, Bolland DJ, Corcoran AE, Fraser P. 2007. Myc dynamically and preferentially relocates to a transcription factory occupied by Igh. PLoS Biol 5: e192.

Parada L, McQueen P, Misteli T. 2004. Tissue-specific spatial organization of genomes. Genome Biology 7: R44.

Parada L, McQueen P, Munson P, Misteli T. 2002. Conservation of relative chromosome positioning in normal and cancer cells. Curr Biol 12: 1692.

Parelho V, Hadjur S, Spivakov M, Leleu M, Sauer S, Gregson HC, Jarmuz A, Canzonetta C, Webster Z, Nesterova T, et al. 2008. Cohesins functionally associate with CTCF on mammalian chromosome arms. Cell 132: 422-433.

Pegoraro G, Kubben L, Wickert U, Goehler H, Hoffman K, Misteli T. 2009. Aging-related chromatin defects via loss of the NURD complex. Nat Cell Biol in press.

Peters JM, Tedeschi A, Schmitz J. 2008. The cohesin complex and its roles in chromosome biology. Genes Dev 22: 3089-3114.

Phillips JE, Corces VG. 2009. CTCF: Master weaver of the genome. Cell 137: 1194-1211.

Pomeroy SL, Tamayo P, Gaasenbeek M, Sturla LM, Angelo M, McLaughlin ME, Kim JY, Goumnerova LC, Black PM, Lau C, et al. 2002. Prediction of central nervous system embryonal tumour outcome based on gene expression. Nature 415: 436-442.

Roix JJ, McQueen PG, Munson PJ, Parada LA, Misteli T. 2003. Spatial proximity of translocation-prone gene loci in human lymphomas. Nat Genet 34: 287-291.

Rollins RA, Morcillo P, Dorsett D. 1999. Nipped-B, a Drosophila homologue of chromosomal adherins, participates in activation by remote enhancers in the cut and Ultrabithorax genes. Genetics 152: 577-593.

Ruginis T, Taglia L, Matusiak D, Lee BS, Benya RV. 2006. Consequence of gastrin-releasing peptide receptor activation in a human colon cancer cell line: A proteomic approach. J Proteome Res 5: 1460-1468. 
Sarg B, Koutzamani E, Helliger W, Rundquist I, Lindner HH. 2002. Postsynthetic trimethylation of histone H4 at lysine 20 in mammalian tissues is associated with aging. J Biol Chem 277: 39195-39201.

Scaffidi P, Misteli T. 2006. Lamin A-dependent nuclear defects in human aging. Science 312: 1059-1063.

Schmidt CK, Brookes N, Uhlmann F. 2009. Conserved features of cohesin binding along fission yeast chromosomes. Genome Biol 10: R52.

Schönherr N, Binder G, Korsch E, Kämmerer E, Wollmann HA, Eggermann T. 2008. Are H19 variants associated with Silver-Russell syndrome? J Pediatr Endocrinol Metab 21: 985-993.

Shen S, Liu A, Li J, Wolubah C, Casaccia-Bonnefil P. 2008. Epigenetic memory loss in aging oligodendrocytes in the corpus callosum. Neurobiol Aging 29: 452-463.

Shumaker DK, Dechat T, Kohlmaier A, Adam SA, Bozovsky MR, Erdos MR, Eriksson M, Goldman AE, Khuon S, Collins FS, et al. 2006. Mutant nuclear lamin A leads to progressive alterations of epigenetic control in premature aging. Proc Natl Acad Sci 103: 8703-8708.

Sinclair DA, Guarente L. 1997. Extrachromosomal rDNA circles-a cause of aging in yeast. Cell 91: 1033-1042.

Soutoglou E, Dorn JF, Sengupta K, Jasin M, Nussenzweig A, Ried T, Danuser G, Misteli T. 2007. Positional stability of single double-strand breaks in mammalian cells. Nat Cell Biol 9: 675-682.

Sparago A, Cerrato F, Vernucci M, Ferrero GB, Silengo MC, Riccio A. 2004. Microdeletions in the human H19 DMR result in loss of IGF2 imprinting and BeckwithWiedemann syndrome. Nat Genet 36: 958-960.

Splinter E, Heath H, Kooren J, Palstra RJ, Klous P, Grosveld F, Galjart N, de Laat W. 2006. CTCF mediates long-range chromatin looping and local histone modification in the $\beta$-globin locus. Genes Dev 20: 2349-2354.

Therman E, Susman B, Denniston C. 1989. The nonrandom participation of human acrocentric chromosomes in Robertsonian translocations. Ann Hum Genet 53: 49-65.
Tonkin ET, Wang TJ, Lisgo S, Bamshad MJ, Strachan T. 2004. NIPBL, encoding a homolog of fungal Scc2-type sister chromatid cohesion proteins and fly Nipped-B, is mutated in Cornelia de Lange syndrome. Nat Genet 36: 636-641.

Vega H, Waisfisz Q, Gordillo M, Sakai N, Yanagihara I, Yamada M, van Gosliga D, Kayserili H, Xu C, Ozono K, et al. 2005. Roberts syndrome is caused by mutations in ESCO2, a human homolog of yeast ECO1 that is essential for the establishment of sister chromatid cohesion. Nat Genet 37: 468-470.

Visser AE, Jaunin F, Fakan S, Aten JA. 2000. High resolution analysis of interphase chromosome domains. J Cell Sci 113: $2585-2593$.

Wendt KS, Yoshida K, Itoh T, Bando M, Koch B, Schirghuber E, Tsutsumi S, Nagae G, Ishihara K, Mishiro T, et al. 2008. Cohesin mediates transcriptional insulation by CCCTC-binding factor. Nature 451: 796-801.

Wilson VL, Jones PA. 1983. DNA methylation decreases in aging but not in immortal cells. Science 220: 1055-1057.

Witcher M, Emerson BM. 2009. Epigenetic silencing of the 16(INK4a) tumor suppressor is associated with loss of CTCF binding and a chromatin boundary. Mol Cell 34: 271-284.

Woodcock CL. 2006. Chromatin architecture. Curr Opin Struct Biol 16: 213-220.

Xie X, Lu J, Kulbokas EJ, Golub TR, Mootha V, Lindblad-Toh K, Lander ES, Kellis M. 2005. Systematic discovery of regulatory motifs in human promoters and 3' UTRs by comparison of several mammals. Nature 434: 338-345.

Yoon YS, Jeong S, Rong Q, Park KY, Chung JH, Pfeifer K. 2007. Analysis of the H19ICR insulator. Mol Cell Biol 27: 3499-3510.

Zhao Z, Tavoosidana G, Sjolinder M, Gondor A, Mariano P, Wang S, Kanduri C, Lezcano M, Sandhu KS, et al. 2006. Circular chromosome conformation capture (4C) uncovers extensive networks of epigenetically regulated intra- and interchromosomal interactions. Nat Genet 38: $1341-1347$. 


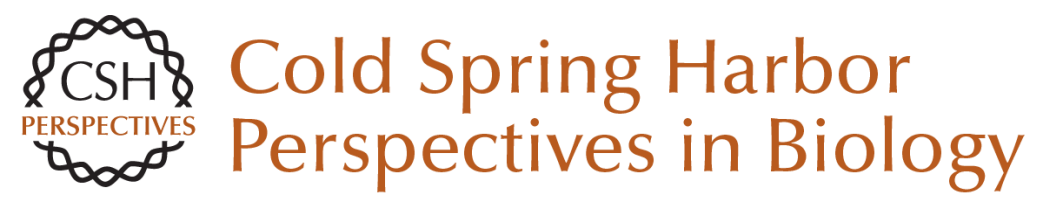

\section{Higher-order Genome Organization in Human Disease}

Tom Misteli

Cold Spring Harb Perspect Biol 2010; doi: 10.1101/cshperspect.a000794 originally published online June 30,2010

\section{Subject Collection The Nucleus}

Nuclear Compartments: An Incomplete Primer to Nuclear Compartments, Bodies, and Genome Organization Relative to Nuclear Architecture Andrew S. Belmont

Uncovering the Principles of Genome Folding by 3D Chromatin Modeling

Asli Yildirim, Lorenzo Boninsegna, Yuxiang Zhan, et al.

3D or Not 3D: Shaping the Genome during Development Juliane Glaser and Stefan Mundlos

The Impact of Space and Time on the Functional Output of the Genome Marcelo Nollmann, Isma Bennabi, Markus Götz, et al.

Chromatin Mechanisms Driving Cancer

Berkley Gryder, Peter C. Scacheri, Thomas Ried, et al.

Liquid-Liquid Phase Separation in Chromatin Karsten Rippe

Mechanical Forces in Nuclear Organization Yekaterina A. Miroshnikova and Sara A. Wickström

Imaging Organization of RNA Processing within the Nucleus

Jeetayu Biswas, Weihan Li, Robert H. Singer, et al.
Mechanisms of Chromosome Folding and Nuclear Organization: Their Interplay and Open Questions Leonid Mirny and Job Dekker

Epigenetic Reprogramming in Early Animal Development

Zhenhai Du, Ke Zhang and Wei Xie

Essential Roles for RNA in Shaping Nuclear Organization

Sofia A. Quinodoz and Mitchell Guttman

The Molecular and Nuclear Dynamics of

$\mathrm{X}$-Chromosome Inactivation

François Dossin and Edith Heard

Structure, Maintenance, and Regulation of

Nuclear Pore Complexes: The Gatekeepers of the

Eukaryotic Genome Marcela Raices and Maximiliano A. D'Angelo

The Nuclear Lamina Xianrong Wong, Ashley J. Melendez-Perez and Karen L. Reddy

The Nuclear Pore Complex as a Transcription Regulator Michael Chas Sumner and Jason Brickner

Physical Nature of Chromatin in the Nucleus Kazuhiro Maeshima, Shiori lida and Sachiko Tamura

For additional articles in this collection, see http://cshperspectives.cshlp.org/cgi/collection/

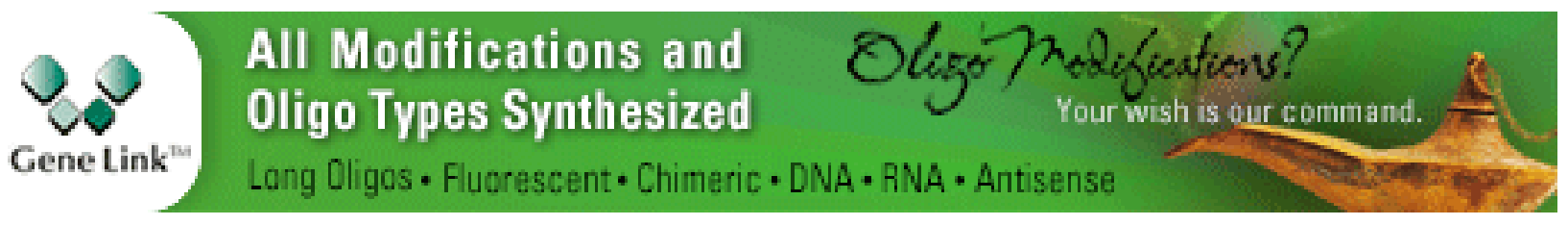


For additional articles in this collection, see http://cshperspectives.cshlp.org/cgi/collection/

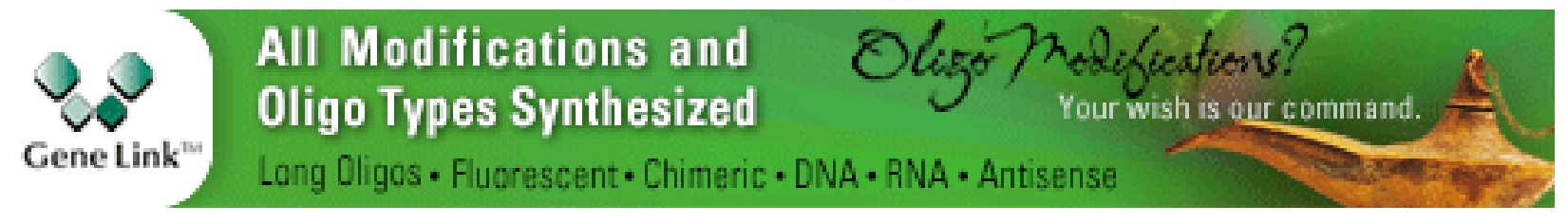

Copyright @ 2010 Cold Spring Harbor Laboratory Press; all rights reserved 